\title{
NON-UNITARY SET-THEORETICAL SOLUTIONS TO THE QUANTUM YANG-BAXTER EQUATION
}

\author{
Alexander Soloviev
}

\begin{abstract}
We develop a theory of non-unitary set-theoretical solutions to the Quantum Yang-Baxter equation. Our results generalize those obtained by Etingof, Schedler and the author in [ESS]. We remark that some of our constructions are similar to constructions obtained by Lu, Yan, and Zhu in [LYZ].
\end{abstract}

\section{Introduction}

In this paper we study set-theoretical solutions to the Quantum Yang-Baxter equation, i.e. permutations $R: X \times X \rightarrow X \times X$ with $X$ being a non-empty set such that

$$
R^{12} R^{13} R^{23}=R^{23} R^{13} R^{12} \text { in } \operatorname{Aut}(X \times X \times X) .
$$

In the above $R^{12}, R^{13}, R^{23} \in \operatorname{Aut}(X \times X \times X)$ stand for $R$ acting in 1,2 ; 1,3 ; and 2,3 components of $X \times X \times X$ correspondingly. The idea to consider set-theoretical solutions first appeared in [Dr]. Later on, Etingof, Schedler and the author [ESS] studied set-theoretical solutions to the Quantum Yang-Baxter equation which satisfied additional properties of unitarity and nondegeneracy (crossing symmetry). In particular, [ESS] contained the classification of nondegenerate unitary set-theoretical solutions to QYBE in group theoretical terms as well as numerous classes of examples of such solutions. Subsequently, Lu, Yan and Zhu in [LYZ] showed that many of the constructions from [ESS] hold in a more general case of nondegenerate but not necessarily unitary set-theoretical solutions to QYBE.

Following [ESS] and [LYZ], we develop a theory of nondegenerate settheoretical solutions to QYBE. Particularly, we show that the unitarity condition that was used in [ESS] for group theoretical characterization of unitary nondegenerate set-theoretical solutions to QYBE can be dropped. We give a group theoretical characterization of general set-theoretical nondegenerate solutions to QYBE in Theorem 2.7. We also introduce injective solutions, study their properties, and show that there is a combinatorial criterion (Theorem 2.9) describing the class of injective nondegenerate solutions to QYBE. Injectivity property, which is a generalization of involutivity, is important for studying affine solutions. In particular the classification of unitary affine solutions given in [ESS] can be generalized to include injective solutions.

Received March 30, 2000. 
It was shown in [ESS] that the structure group of a nondegenerate unitary set-theoretical solution to QYBE on a set with $\mathrm{N}$ elements always has an abelian subgroup of finite index and of rank N. We compute (Theorem 2.10) the rank of a finite index abelian subgroup of the structure group for an arbitrary nondegenerate finite solution and show that this rank never exceeds $\mathrm{N}$, with the equality taking place only in the unitary case.

In the second part of the paper we discuss the applications of the developed theory to examples. In particular, we classify affine solutions on an abelian group. It is proved that injective affine solutions are obtained from the representations of the algebra generated by invertible elements $p, q$, and $z$ are subject to $p q=q p$ and $z^{2}-z(p+q)+p q=0$.

\section{Structure groups}

2.1. Construction of the structure groups. Let $X$ be a nonempty set and $S: X \times X \rightarrow X \times X$ a bijective map. We call a pair $(X, S)$ a braided set if the following braiding condition holds in $X \times X \times X$ :

$$
S_{1} S_{2} S_{1}=S_{2} S_{1} S_{2}
$$

where $S_{1}=S \times i d, S_{2}=i d \times S$.

Remark. Consider the map $R: X \times X \rightarrow X \times X$ given by $R=\sigma S$, where $\sigma(x, y)=(y, x)$ for $x, y \in X$. Then $(X, S)$ is a braided set if and only if $R$ satisfies the Quantum Yang-Baxter equation.

It is useful to associate with a braided set $(X, S)$ two groups $G_{X}$ and $A_{X}$.

Definition 1. Define the group $G_{X}$ as the group generated by the elements of $X$ subject to the relations $x y=y_{1} x_{1}$ if $S(x, y)=\left(y_{1}, x_{1}\right)$, where $x, y \in X$. We call $G_{X}$ the structure group of the braided set $(X, S)$.

Definition 2. Define the group $A_{X}$ as the group generated by elements of $X$ subject to relations $x_{1} \bullet y=y_{2} \bullet x_{1}$, where $x, y \in X$ and $x_{1}, y_{2} \in X$ are defined out of relations $S(x, y)=\left(y_{1}, x_{1}\right), S\left(y_{1}, x_{1}\right)=\left(x_{2}, y_{2}\right)$. We call $A_{X}$ the derived structure group of the braided set $(X, S)$.

We introduce the maps $g: X \times X \rightarrow X$ and $f: X \times X \rightarrow X$ as components of $S$, i.e. for $x, y \in X$,

$$
S(x, y)=\left(g_{x}(y), f_{y}(x)\right)
$$

Definition 3. (i) We call a set $(X, S)$ nondegenerate if $g_{x}(y)$ is a bijective function of $y$ for fixed $x$ and $f_{y}(x)$ is a bijective function of $x$ for fixed $y$. (ii) $W e$ call a set $(X, S)$ involutive if $S^{2}=i d_{X^{2}}$. A braided set which is involutive will be called symmetric.

In particular, for a symmetric set $(X, S)$ we see that the group $A_{X}$ is the free abelian group generated by elements of $X$. Note that the properties of involutivity and nondegeneracy are equivalent to corresponding properties of unitarity and crossing symmetry for the map $R=\sigma S$ [ESS]. 
Recall that the braid group $B_{n}$ for $n \geq 2$ is generated by elements $b_{i}, 1 \leq$ $i \leq n-1$, with defining relations

$$
b_{i} b_{j}=b_{j} b_{i},|i-j|>1, b_{i} b_{i+1} b_{i}=b_{i+1} b_{i} b_{i+1}
$$

and recall that the symmetric group $S_{n}$ is the quotient of $B_{n}$ by the relations $b_{i}^{2}=1$. The following obvious proposition explains our terminology. Let $S_{n}^{i i+1}$ : $X^{n} \rightarrow X^{n}$ be defined as $S_{n}^{i i+1}=i d_{X^{i-1}} \times S \times i d_{X^{n-i-1}}$.

Theorem 2.1 ([ESS]). (i) The assignment $b_{i} \rightarrow S_{n}^{i i+1}$ extends to an action of $B_{n}$ on $X^{n}(n \geq 3)$ if and only if $(X, S)$ is a braided set.

(ii) The assignment $b_{i} \rightarrow S_{n}^{i i+1}$ extends to an action of $S_{n}$ on $X^{n}(n \geq 3)$ if and only if $(X, S)$ is a symmetric set.

Definition 4. The action of Theorem 2.1 is called the twisted action of $B_{n}$ (or $\left.S_{n}\right)$ given by $S$.

The following result shows how a nondegenerate braided set $(X, S)$ gives rise to two actions of the structure group $G_{X}$ on the set $X$.

Theorem 2.2. ([ESS]) Suppose that $(X, S)$ is nondegenerate. Then $(X, S)$ is a braided set if and only if the following conditions are simultaneously satisfied:

(i) the assignment $x \rightarrow f_{x}$ is a right action of $G_{X}$ on $X$;

(ii) the assignment $x \rightarrow g_{x}$ is a left action of $G_{X}$ on $X$;

(iii) the linking relation

$$
f_{g_{f_{y}(x)}(z)}\left(g_{x}(y)\right)=g_{f_{g_{y}(z)}(x)}\left(f_{z}(y)\right)
$$

holds.

Proof. Conditions (i)-(iii) are exactly the three components of the braid relation (2.1).

In light of the above proposition it makes sense to introduce the notations $x \circ y=g_{x}(y)$ and $y * x=f_{y}^{-1}(x)$ for $x, y \in X$. Then if $(X, S)$ is a nondegenerate braided set, we can extend $*$ and $\circ$ to left actions of $G_{X}$ on $X$. We will denote the actions of an element $g \in G_{X}$ on an element $x \in X$ by $g \circ x$ and $g * x$ correspondingly.

From now on we always assume $(X, S)$ to be a nondegenerate braided set. Sometimes we refer to nondegenerate braided sets as to "solutions", meaning that $S$ is a solution to braid equation (2.1).

Define $\phi: X \times X \rightarrow X$ by

$$
\phi(y, x)=x^{-1} *((y * x) \circ y),
$$

and $S^{\prime}: X \times X \rightarrow X \times X$ as $S^{\prime}(x, y)=(\phi(y, x), x)$.

Theorem 2.3. (i) $\phi(y, z)$ is $G_{X}$-invariant w.r.t. *action, i.e. for $g \in G_{X}$, $g * \phi(y, z)=\phi(g * y, g * z)$.

(ii) $\left(X, S^{\prime}\right)$ is a nondegenerate braided set. We call this set the derived braided set or the derived solution.

(iii) The structure group of the derived solution is the derived structure group. 
(iv) For each integer $n \geq 2$ there exists a bijection $J_{n}: X^{n} \rightarrow X^{n}$ such that $J_{n} S_{n}^{i i+1} J_{n}^{-1}=\left(S^{\prime}\right)_{n}^{i i+1}$, where $S_{n}^{i i+1}$ is the same as in Theorem 2.1. In this way, twisted actions of $B_{n}$ given by $S$ and $S^{\prime}$ are conjugate.

We remark that the statement (iv) of Theorem 2.3 was proved in [ESS] (cf. Prop. 1.7) for unitary solutions and in [LYZ] (cf. Th. 3) for injective solutions that are defined in Section 2.3.

Proof. It is easy to see that (iv) implies (ii). Statement (iii) follows from definitions of structure group, derived structure group and $\phi$. Let us show that (i) implies (iv). Define $J_{n}$ inductively as $J_{1}=i d_{X}, J_{n}=Q_{n}\left(J_{n-1} \times i d_{X}\right)$, where $Q_{n}: X^{n} \rightarrow X^{n}$ is defined as $Q_{n}\left(x_{1}, \ldots, x_{n}\right)=\left(x_{n}^{-1} * x_{1}, \ldots, x_{n}^{-1} * x_{n-1}, x_{n}\right)$. We prove formula $J_{n} S_{n}^{i i+1} J_{n}^{-1}=\left(S^{\prime}\right)_{n}^{i i+1}$ by induction on $n$. For $n=2$ (induction base) the relation follows directly from definition of $\phi$. Suppose the relation holds for $n=k$, let us prove it holds for $n=k+1$. Since $J_{k+1}=Q_{k+1}\left(J_{k} \times i d_{X}\right)$ and $Q_{k+1}$ commutes with $\left(S^{\prime}\right)_{k+1}^{i i+1}$ for $i<k$ (by (i)) the relation is true for $i<k$. So, it remains to prove it for $i=k$ when it becomes identical to the relation of the induction base.

Now we have to show that (i) is true. It is enough to check that for every $t, y, z \in X, t^{-1} * \phi(y, z)=\phi\left(t^{-1} * y, t^{-1} * z\right)$ or, equivalently by $(2.2)$ :

$$
\begin{aligned}
& t^{-1} *\left(z^{-1} *((y * z) \circ y)\right)= \\
&\left(t^{-1} * z\right)^{-1} *\left(\left(\left(t^{-1} * y\right) *\left(t^{-1} * z\right)\right) \circ\left(t^{-1} * y\right)\right) .
\end{aligned}
$$

The linking relation of Theorem 2.2 states that for $x, y, t \in X$

$$
\left(\left(y^{-1} * x\right) \circ t\right)^{-1} *(x \circ y)=\left((y \circ t)^{-1} * x\right) \circ\left(t^{-1} * y\right)
$$

holds. If we substitute $x=y * z$ in relation (2.4) we can rewrite its right hand side as follows:

$$
\left((y \circ t)^{-1} *(y * z)\right) \circ\left(t^{-1} * y\right)=\left((y \circ t)^{-1} *\left(y t *\left(t^{-1} * z\right)\right)\right) \circ\left(t^{-1} * y\right)
$$

where the product $y t$ is the product of elements $y, t$ in group $G_{X}$, thus $y t=$ $(y \circ t)\left(t^{-1} * y\right)$ and

$$
\left((y \circ t)^{-1} *\left(y t *\left(t^{-1} * z\right)\right)\right) \circ\left(t^{-1} * y\right)=\left(\left(t^{-1} * y\right) *\left(t^{-1} * z\right)\right) \circ\left(t^{-1} * y\right) .
$$

In this way, relation (2.4) is equivalent to relation (2.5) below:

$$
(z \circ t)^{-1} *((y * z) \circ y)=\left(\left(t^{-1} * y\right) *\left(t^{-1} * z\right)\right) \circ\left(t^{-1} * y\right) .
$$

Substituting, (2.5) into the right side of (2.3) we get

$$
\left(t^{-1} * z\right)^{-1} *\left(\left(\left(t^{-1} * y\right) *\left(t^{-1} * z\right)\right) \circ\left(t^{-1} * y\right)\right)=\left(\left(t^{-1} * z\right)^{-1}(z \circ t)^{-1}\right) *((y * z) \circ y) \text {. }
$$

Using the relation $t^{-1} z^{-1}=\left(t^{-1} * z\right)^{-1}(z \circ t)^{-1}$ in group $G_{X}$ we verify that invariance relation (2.3) is true. The theorem is proved.

Note that by construction we have two natural maps $\psi_{G}: X \rightarrow G_{X}$ and $\psi_{A}: X \rightarrow A_{X}$ that are not necessarily embeddings (see Examples below). Theorem 2.4 shows that the latter map is $G_{X}$-invariant with respect to a suitable $G_{X}$-module structure on $A_{X}$. Define the group Permut $(X)$ as the group of all 
permutations of the set $X$. Then both $*$ and $\circ$ define homomorphisms from $G_{X}$ to Permut $(X)$. Denote by $\operatorname{Aut}_{X}\left(A_{X}\right)$ the group of automorphisms of $A_{X}$ that map the generating set $\psi_{A}(X)$ onto itself.

Theorem 2.4. For a nondegenerate braided set $(X, S)$ the group homomorphism * $: G_{X} \rightarrow \operatorname{Permut}(X)$ can be uniquely lifted to the homomorphism $\hat{*}: G_{X} \rightarrow \operatorname{Aut}_{X}\left(A_{X}\right)$ such that for any $g \in G_{X}, x \in X \psi_{A}(g * x)=g \hat{*} \psi_{A}(x)$.

Proof. It is clear that the map $*: G_{X} \rightarrow \operatorname{Permut}(X)$ can be uniquely extended to the homomorphism from the group $G_{X}$ to the group of automorphisms of the free group generated by $X$. So, it is enough to show that such an extension respects the generating relations in group $A_{X}$. This immediately follows from statements (i), (iii) of Theorem 2.3.

Example [Dr]. Let $X$ be a union of conjugacy classes in a group $G$, i.e. $g X g^{-1}=$ $X$ for any $g \in G$. Define $S: X \times X \rightarrow X \times X$ as $S(x, y)=\left(x y x^{-1}, x\right)$ for $x, y \in X$. It is easy to see that $(X, S)$ is a braided nondegenerate set. It was called the conjugate solution in [LYZ]. Clearly, this solution coincides with its own derived solution - therefore $G_{X}=A_{X}$. The action of $G_{X}$ on $A_{X}$ is easily seen to be trivial, i.e. $g \hat{*} h=h$ for any $g \in G_{X}, h \in A_{X}$. The embedding $i: X \rightarrow G$ can be extended to a homomorphism $I: G_{X} \rightarrow G$ that maps elements of $\psi_{G}(X)$ onto $X$, thus the map $\psi_{G}: X \rightarrow G_{X}$ is injective. So $(X, S)$ is an injective solution (cf. Definition 8).

2.2. Bijective 1-cocycle. Recall that we started with a nondegenerate braided set $(X, S)$ and constructed two groups $G_{X}, A_{X}$ and the action $\hat{*}$ of $G_{X}$ on $A_{X}$. In the future we drop ^ in $\hat{*}$ and denote the action of $g \in G_{X}$ on $h \in A_{X}$ from Theorem 2.4 simply by $g * h$. Theorem 2.5 is the main step towards classification of nondegenerate braided sets in group theoretical terms.

Definition 5. Suppose a group $G$ acts on a group A by automorphisms meaning that there is a homomorphism from $G$ to $\operatorname{Aut}(A)$. Denote the product of elements $g_{1}, g_{2}$ in $G$ by $g_{1} g_{2}$ and the product of elements $a_{1}, a_{2}$ in $A$ by $a_{1} \bullet a_{2}$. We call a map $\pi: G \rightarrow A$ a 1-cocycle if for any $g_{1}, g_{2} \in G$

$$
\pi\left(g_{1} g_{2}\right)=\left(g_{2}^{-1} * \pi\left(g_{1}\right)\right) \bullet \pi\left(g_{2}\right)
$$

where $*$ stands for the action of $G$ on $A$.

Theorem 2.5. (cf. [LYZ], Th. 2 and [ESS], Prop. 2.5) For a nondegenerate braided set $(X, S)$ there exists a unique bijective 1-cocycle $\pi: G_{X} \rightarrow A_{X}$ such that $\pi \psi_{G}=\psi_{A}$ on $X$.

Remark. In [LYZ] the authors introduced on $G_{X}$ another group structure. It follows from our and their results that $G_{X}$ with the new group structure is isomorphic to $A_{X}$ via $\pi: G_{X} \rightarrow A_{X}$.

\section{Proof. Construction of the 1-cocycle.}


Let us construct the 1-cocycle $\pi: G_{X} \rightarrow A_{X}$. Consider the semidirect product $G_{X} \ltimes A_{X}$. The group $G_{X} \ltimes A_{X}$ consists of pairs $(g, h), g \in G_{X}, h \in A_{X}$ with the group operation given by the formula

$$
(g, h)\left(g^{\prime}, h^{\prime}\right)=\left(g g^{\prime},\left(\left(g^{\prime}\right)^{-1} * h\right) \bullet h^{\prime}\right) .
$$

Define the map $s: X \rightarrow G_{X} \ltimes A_{X}$ via the formula $s(x)=\left(\psi_{G}(x), \psi_{A}(x)\right)$ for any $x \in X$. We claim that there exists a group homomorphism $\bar{s}: G_{X} \rightarrow G_{X} \ltimes A_{X}$ such that $\bar{s} \psi_{G}=s$. Indeed, since $\psi_{G}(X)$ generates the whole $G_{X}$ to show that $\bar{s}$ exists it is necessary and sufficient to check that $s$ respects the relations in $G_{X}$, i.e. that for $x, y \in X s(x) s(y)$ is equal to $s(x \circ y) s\left(y^{-1} * x\right)$ in group $G_{X} \ltimes A_{X}$. When we formally multiply terms out, the above condition transforms into the relation

$$
\left(x y,\left(y^{-1} * x\right) \bullet y\right)=\left((x \circ y)\left(y^{-1} * x\right),\left(\left(y^{-1} * x\right)^{-1} *(x \circ y)\right) \bullet\left(y^{-1} * x\right)\right),
$$

which coincides componentwise with the defining relations in groups $G_{X}, A_{X}$. Let us define the projection $p: G_{X} \ltimes A_{X} \rightarrow A_{X}$ by the formula $p(g, h)=h$. Introduce $\pi: G_{X} \rightarrow A_{X}$ as $\pi=p \bar{s}$. Clearly, $\pi$ is a 1-cocycle. The tricky part is to show that $\pi$ is bijective.

Proof that $\pi$ is bijective.

Lemma 1. (i) For $x_{1}, x_{2} \in X, g \in G_{X}$ if $\psi_{G}\left(x_{1}\right)=\psi_{G}\left(x_{2}\right)$ then $\psi_{G}\left(g * x_{1}\right)=$ $\psi_{G}\left(g * x_{2}\right)$ and $\psi_{G}\left(g \circ x_{1}\right)=\psi_{G}\left(g \circ x_{2}\right)$.

(ii) For $x \in X \psi_{G}((x * x) \circ x)=\psi_{G}(x * x)$ and $\psi_{G}\left(\left(x^{-1} \circ x\right)^{-1} * x\right)=\psi_{G}\left(x^{-1} \circ x\right)$.

(iii) For $x \in X \psi_{G}(x)=\psi_{G}\left((x * x)^{-1} \circ(x * x)\right)=\psi_{G}\left(\left(x^{-1} \circ x\right) *\left(x^{-1} \circ x\right)\right)$.

To prove the lemma we notice that $S(x * x, x)=((x * x) \circ x, x)$ therefore $\psi_{G}((x * x) \circ x) \psi_{G}(x)=\psi_{G}(x * x) \psi_{G}(x)$ and $\psi_{G}((x * x) \circ x)=\psi_{G}(x * x)$. Similarly $S\left(x, x^{-1} \circ x\right)=\left(x,\left(x^{-1} \circ x\right)^{-1} * x\right)$ implies that $\psi_{G}\left(\left(x^{-1} \circ x\right)^{-1} * x\right)=\psi_{G}\left(x^{-1} \circ x\right)$. So statement (ii) of the lemma is proved. It is clear that statement (iii) follows from (i) and (ii) thus it remains to prove (i).

Let us show that $\psi_{G}\left(x_{1}\right)=\psi_{G}\left(x_{2}\right)$ implies that $\psi_{G}\left(g * x_{1}\right)=\psi_{G}\left(g * x_{2}\right)$. For a fixed $z \in X$ it is enough to reason that $\psi_{G}\left(x_{1}\right)=\psi_{G}\left(x_{2}\right)$ if and only if $\psi_{G}\left(z^{-1} * x_{1}\right)=\psi_{G}\left(z^{-1} * x_{2}\right)$. Since $S\left(x_{1}, z\right)=\left(x_{1} \circ z, z^{-1} * x_{1}\right)$ and $S\left(x_{2}, z\right)=$ $\left(x_{2} \circ z, z^{-1} * x_{2}\right)$ we see that

$$
\psi_{G}\left(x_{1}\right) \psi_{G}(z)=\psi_{G}\left(x_{1} \circ z\right) \psi_{G}\left(z^{-1} * x_{1}\right)
$$

and

$$
\psi_{G}\left(x_{2}\right) \psi_{G}(z)=\psi_{G}\left(x_{2} \circ z\right) \psi_{G}\left(z^{-1} * x_{2}\right) .
$$

Suppose $\psi_{G}\left(x_{1}\right)=\psi_{G}\left(x_{2}\right)$ then since $*$ is an action of $G_{X}$ on $X$ we immediately see that $\psi_{G}\left(x_{1} \circ z\right)=\psi_{G}\left(x_{2} \circ z\right)$ therefore equations (2.7)-(2.8) imply that $\psi_{G}\left(z^{-1} * x_{1}\right)=\psi_{G}\left(z^{-1} * x_{2}\right)$. Conversely, assume that $\psi_{G}\left(z^{-1} * x_{1}\right)=$ $\psi_{G}\left(z^{-1} * x_{2}\right)$. Our task is to prove that $\psi_{G}\left(x_{1}\right)=\psi_{G}\left(x_{2}\right)$. We use the statement (iv) of Theorem 2.3 in the following form: $S=J_{2}^{-1} S^{\prime} J_{2}$, where $J_{2}(x, y)=\left(y^{-1} * x, y\right)$. We notice that $S^{\prime} J_{2}\left(x_{1}, z\right)=\left(\phi\left(z, z^{-1} * x_{1}\right), z^{-1} * x_{1}\right)$ and $S^{\prime} J_{2}\left(x_{2}, z\right)=\left(\phi\left(z, z^{-1} * x_{2}\right), z^{-1} * x_{2}\right)$. Since $\phi\left(z, z^{-1} * x_{1}\right)$ is the action 
of $\psi_{A}\left(z^{-1} * x_{1}\right) \in A_{X}$ on $z \in X$ and $\psi_{A}=\pi \psi_{G}$ we see that $\phi\left(z, z^{-1} * x_{1}\right)=$ $\phi\left(z, z^{-1} * x_{2}\right)$. In this way, first components of $S\left(x_{1}, z\right)=J_{2}^{-1} S^{\prime} J_{2}\left(x_{1}, z\right)$ and $S\left(x_{2}, z\right)=J_{2}^{-1} S^{\prime} J_{2}\left(x_{2}, z\right)$ coincide, i.e. $x_{1} \circ z=x_{2} \circ z$. So the equations (2.7)-(2.8) imply that $\psi_{G}\left(x_{1}\right)=\psi_{G}\left(x_{2}\right)$. In a similar fashion one can show that $\psi_{G}\left(x_{1}\right)=\psi_{G}\left(x_{2}\right)$ if and only if $\psi_{G}\left(z \circ x_{1}\right)=\psi_{G}\left(z \circ x_{2}\right)$. The lemma is proved.

We aim to construct the map $h: A_{X} \rightarrow G_{X}$ inverse to $\pi$. At first we define $h$ on $F_{X}$ - the free group generated by $X$ and then show that it descends to $A_{X}$. We note that $G_{X}$ acts on $F_{X}$ via $f \rightarrow g * f$ for $f \in F_{X}, g \in G_{X}$ - the action induced from *action of $G_{X}$ on $X$. For $x \in X \subset F_{X}$ define

$$
h(x)=\psi_{G}(x), h\left(x^{-1}\right)=\left(\psi_{G}\left(x^{-1} \circ x\right)\right)^{-1} .
$$

Notice that $x^{-1} \circ x$ is the same as $\psi_{G}(x)^{-1} \circ x$. For an element $f=x_{1} \bullet \ldots \bullet x_{k} \in F_{X}$ of length $k$ define inductively $h\left(x_{1} \bullet \ldots \bullet x_{k}\right)=h\left(h\left(x_{2} \bullet \ldots \bullet x_{k}\right) * x_{1}\right) h\left(x_{2} \bullet \ldots \bullet x_{k}\right)$, where for each $i, x_{i} \in X \subset F_{X}$ or $x_{i}^{-1} \in X \subset F_{X}$. In the above $f=x_{1} \bullet \ldots \bullet x_{k}$ was the minimum decomposition of $f \in F_{X}$ and $k$, the length of $\mathrm{f}$, is the number of elements in such a decomposition. The only element of length 0 in $F_{X}$ is identity $e$ and we put $h(e)=1 \in G_{X}$. We claim that for $a, b \in F_{X}$

$$
h(a \bullet b)=h(h(b) * a) h(b) .
$$

Indeed, we can verify (2.9) by induction on the length of $a$. Suppose we know that (2.9) holds for for all elements $a$ of length $k$ and want to check that $h(a \bullet$ $y \bullet b)=h(h(b) *(a \bullet y)) h(b)$ for $y \in X \subset F_{X}$ or $y^{-1} \in X \subset F_{X}$. We simplify $h(h(b) *(a \bullet y)) h(b)$ as follows:

$$
\begin{aligned}
h(h(b) *(a \bullet y)) h(b) & =h((h(b) * a) \bullet(h(b) * y)) h(b) \\
& =h(h(h(b) * y) *(h(b) * a)) h(h(b) * y) h(b) .
\end{aligned}
$$

On the other hand, we know that

$$
\begin{aligned}
h(a \bullet y \bullet b)=h(h(y \bullet b) * a) h(y \bullet b) & =h(h(h(b) * y) h(b) * a) h(h(b) * y) h(b) \\
& =h(h(h(b) * y) *(h(b) * a)) h(h(b) * y) h(b) .
\end{aligned}
$$

We see that $h(a \bullet y \bullet b)=h(h(b) *(a \bullet y)) h(b)$. In this way, we just need to check the induction base, namely that $h(y \bullet b)=h(h(b) * y) h(b)$. There are two cases to consider:

1. Length $(y \bullet b)=1+$ Length $(b)$.

2. Length $(y \bullet b)=$ Length $(b)-1$, i.e. $b=y^{-1} \bullet b^{\prime}$ and Length $(b)=$ Length $\left(b^{\prime}\right)+1$.

In the first case the formula $h(y \bullet b)=h(h(b) * y) h(b)$ follows from definition of $h$. In the second case without loss of generality assume that $y \in X$. Then, 


$$
\begin{aligned}
& h(b)=h\left(y^{-1} \bullet b^{\prime}\right)=h\left(\left(h\left(b^{\prime}\right) * y\right)^{-1}\right) h\left(b^{\prime}\right) \text { and letting } h\left(b^{\prime}\right) * y=z \text { we get } \\
& \quad \begin{array}{l}
h(h(b) * y) h(b)=h\left(h\left(\left(h\left(b^{\prime}\right) * y\right)^{-1}\right) h\left(b^{\prime}\right) * y\right) h\left(\left(h\left(b^{\prime}\right) * y\right)^{-1}\right) h\left(b^{\prime}\right) \\
\left.=h\left(h\left(z^{-1}\right) * z\right)\right) h\left(z^{-1}\right) h\left(b^{\prime}\right)=\psi_{G}\left(\psi_{G}\left(z^{-1} \circ z\right)^{-1} * z\right) \psi_{G}\left(z^{-1} \circ z\right)^{-1} h\left(b^{\prime}\right) \\
\quad=\left(\text { by Lemma) } \psi _ { G } \left(\left(z^{-1} \circ z\right) \psi_{G}\left(z^{-1} \circ z\right)^{-1} h\left(b^{\prime}\right)=h\left(b^{\prime}\right)=h(y \bullet b) .\right.\right.
\end{array}
\end{aligned}
$$

Now we are going to check that $h$ descends to $A_{X}$. Recall that $A_{X}$ is given by generators - elements of $X$ and relations $\left(z^{-1} *((y * z) \circ y)\right) \bullet z=z \bullet y$ for each $y, z \in X$. We see that

$$
h\left(\left(z^{-1} *((y * z) \circ y)\right) \bullet z\right)=\psi_{G}((y * z) \circ y) h(z)=h(z \bullet y) .
$$

If we define $p(y, z)=\left(z^{-1} *((y * z) \circ y)\right) \bullet z \in F_{X}$ and $q(y, z)=z \bullet y \in F_{X}$ then for any $g \in G_{X}, h\left(g *\left(p(y, z)^{-1} \bullet q(y, z)\right)\right)=h\left(g *\left(q(y, z)^{-1} \bullet p(y, z)\right)\right)=1$.

Indeed, we checked in the proof of Theorem 2.4 that the defining relations of group $A_{X}$ are $G_{X}$-invariant with respect to * action, i.e. $g * p(y, z)=p(g * y, g * z)$ and $g * q(y, z)=q(g * y, g * z)$. Formula $(2.10)$ states that $h(p(y, z))=h(q(y, z))$, therefore

$$
\begin{aligned}
h\left(p(y, z)^{-1}\right. & \bullet q(y, z))=h\left(h(q(y, z)) * p(y, z)^{-1}\right) h(q(y, z)) \\
& =h\left(h(p(y, z)) * p(y, z)^{-1}\right) h(p(y, z))=h\left(p(y, z)^{-1} \bullet p(y, z)\right)=1 .
\end{aligned}
$$

Thus $h\left(g *\left(p(y, z)^{-1} \bullet q(y, z)\right)\right)=h\left(p(g * y, g * z)^{-1} \bullet q(g * y, g * z)\right)=1$. Similarly, $h\left(g *\left(q(y, z)^{-1} \bullet p(y, z)\right)\right)=1$.

In order to check that $h: F_{X} \rightarrow G_{X}$ descends to the map $h: A_{X} \rightarrow G_{X}$ it is enough to check that for any $a, b \in F_{X}, y, z \in X h\left(a \bullet p(y, z)^{-1} \bullet q(y, z) \bullet b\right)=$ $h\left(a \bullet q^{-1}(y, z) \bullet p(y, z) \bullet b\right)=h(a \bullet b)$ holds. By formula $(2.9)$

$$
\begin{aligned}
h\left(a \bullet p^{-1}(y, z) \bullet q(y, z) \bullet b\right) & = \\
& h\left(h\left(p(y, z)^{-1} \bullet q(y, z) \bullet b\right) * a\right) h\left(p(y, z)^{-1} \bullet q(y, z) \bullet b\right),
\end{aligned}
$$

so it is enough to show that $h\left(p^{-1}(y, z) \bullet q(y, z) \bullet b\right)=h(b)$. But $h\left(p^{-1}(y, z) \bullet\right.$ $q(y, z) \bullet b)=h\left(h(b) *\left(p^{-1}(y, z) \bullet q(y, z)\right)\right) h(b)=h(b)$ holds. Similar reasoning allows to check that $h\left(a \bullet q^{-1}(y, z) \bullet p(y, z) \bullet b\right)=h(a \bullet b)$. In this way we constructed the map $h: A_{X} \rightarrow G_{X}$ such that

1. $h(a \bullet b)=h(h(b) * a) h(b)$,

2. $h \psi_{A}=\psi_{G}$.

Condition 1 above implies that both $\pi h: A_{X} \rightarrow A_{X}$ and $h \pi: G_{X} \rightarrow G_{X}$ are group homomorphisms while Condition 2 implies that these homomorphisms are identities if restricted to corresponding generating sets $\psi_{A}(X), \psi_{G}(X)$, thus $\pi h=i d_{A_{X}}, h \pi=i d_{G_{X}}$. Theorem 2.5 is proved.

It turns out then that the groups $G_{X}$ and $A_{X}$ both have abelian subgroups of finite index when $X$ is finite. Namely, define $\Gamma=\left\{g \in G_{X} \mid g * x=x, g \circ x=\right.$ $x$ for all $x \in X\}$. In other words, $\Gamma$ is the intersection of the kernels of left and right actions from Theorem 2.2. 
Theorem 2.6. (cf. Section 2.5 in [ESS] and Prop. 6 in [LYZ])

(i) $\pi(\Gamma)$ is a normal $G_{X}$-invariant (w.r.t. to *-action) subgroup lying in the center of $A_{X} . \Gamma$ is a normal abelian subgroup in $G_{X}$, and $\pi: \Gamma \rightarrow \pi(\Gamma)$ is an isomorphism.

(ii) The 1-cocycle $\pi: G_{X} \rightarrow A_{X}$ can be factored out through $\Gamma$ giving rise to the bijective 1-cocycle $\bar{\pi}: G_{X} / \Gamma \rightarrow A_{X} / \pi(\Gamma)$.

(iii) If $X$ is finite then both $G_{X} / \Gamma$ and $A_{X} / \pi(\Gamma)$ are finite groups.

Proof. (i) Let us show that $\pi(\Gamma)$ is $G_{X}$-invariant and central subgroup in $A_{X}$. From the defining relations in $G_{X}$ we see that $\psi_{G}(x \circ y)=$ $\psi_{G}(x) \psi_{G}(y) \psi_{G}\left(y^{-1} * x\right)^{-1}$ for all $x, y \in X$. In fact, one can easily check that the above formula can be generalized for $x \in G_{X}, y \in X$.

Lemma 2. For $g \in G_{X}, y \in X$,

$$
\psi_{G}(g \circ y)=g \psi_{G}(y) \pi^{-1}\left(\psi_{G}(y)^{-1} * \pi(g)\right)^{-1} .
$$

Proof of Lemma 2. Let us show that if the statement of the Lemma holds for some $g \in G_{X}$ then it holds for $g^{-1}$. We need to check that

$$
g^{-1} \psi_{G}(g \circ y) \pi^{-1}\left(\psi_{G}(g \circ y)^{-1} * \pi\left(g^{-1}\right)\right)^{-1}=y .
$$

Notice that $\pi\left(g^{-1}\right)=(g * \pi(g))^{-1}$ therefore

$$
\psi_{G}(g \circ y)^{-1} * \pi\left(g^{-1}\right)=\left(\left(\psi_{G}(g \circ y)^{-1} g\right) * \pi(g)\right)^{-1} .
$$

Since the statement of the Lemma holds for $g$, we have

$$
\begin{array}{r}
\pi^{-1}\left(\left(\psi_{G}(g \circ y)^{-1} g\right) * \pi(g)\right)^{-1} \\
=\pi^{-1}\left(\left(\pi^{-1}\left(\psi_{G}(y)^{-1} * \pi(g)\right) \psi_{G}(y)^{-1}\right) * \pi(g)\right)^{-1} \\
=\pi^{-1}\left(\pi^{-1}\left(\psi_{G}(y)^{-1} * \pi(g)\right) *\left(\psi_{G}(y)^{-1} * \pi(g)\right)\right)^{-1} \\
=\pi^{-1}\left(\psi_{G}(y)^{-1} * \pi(g)\right) .
\end{array}
$$

This implies the validity of equation (2.11). Similar, a simple computation shows that if the statement of the lemma is true for $g=g_{1}$ and $g=g_{2}$ then it is true for $g=g_{1} g_{2}$. In this way since by definition of $G_{X}$ the lemma is true for $g \in \psi_{G}(X)$ we have proved the lemma. Thus,

$$
y^{-1} g y=\pi^{-1}\left(y^{-1} * \pi(g)\right) .
$$

The condition (2.12) implies that $\pi \Gamma$ belongs to the center of $A_{X}$. Indeed, applying $\pi$ to relation $y^{-1} g=\pi^{-1}\left(y^{-1} * \pi(g)\right) y^{-1}$ we get that $\pi\left(y^{-1}\right)$ commutes with $\pi(g)$. Moreover, for $g \in \Gamma, h \in G_{X}$ 1-cocycle condition implies that

$$
\pi^{-1}(\pi(h) \bullet \pi(g))=h g .
$$

So, in particular the product of elements in $\pi(\Gamma)$ is in $\pi(\Gamma)$ and $\pi$ restricted to $\Gamma$ becomes an isomorphism between $\Gamma$ and $\pi(\Gamma)$.

(ii) The relation (2.13) shows that $\pi$ can be lifted to $\bar{\pi}: G_{X} / \Gamma \rightarrow A_{X} / \pi(\Gamma)$. 
(iii) The kernels of each of the actions $*$, $\circ$ are of finite indexes since the corresponding quotients are isomorphic to subgroups in $\operatorname{Permut}(X)$. So, the intersection of kernels, i.e. subgroup $\Gamma$ has finite index as well.

Definition 6. We call a 7-tuple

$$
\left(G, A, X, \rho_{G A}, \rho_{G A X}, \bar{\pi}, \overline{\psi_{A}}\right)
$$

a bijective cocycle 7-tuple if $G, A$ are groups, $\rho_{G A}: G \rightarrow \operatorname{Aut}(A)$ is an action of $G$ on $A, \rho_{G A X}: G \ltimes A \rightarrow \operatorname{Permut}(X)$ is an action of $G \ltimes A$ on $X, \bar{\pi}: G \rightarrow A$ is a bijective 1-cocycle, $\overline{\psi_{A}}: X \rightarrow A$ is $G \ltimes A$-equivariant, where $G \ltimes A$ acts on A by conjugation.

Note that the action of $G \ltimes A$ gives rise to two actions $\rho_{G X}: G \rightarrow \operatorname{Permut}(X)$ and $\rho_{A X}: A \rightarrow \operatorname{Permut}(X)$. Starting with a bijective cocycle 7 -tuple, let $*$ : $X \times X \rightarrow X$ and $\phi: X \times X \rightarrow X$ be defined as $y * x=\rho_{G X}\left(\overline{\psi_{G}}(y)\right)(x)$ and $\phi(x, y)=\rho_{A X}\left(\overline{\psi_{A}}(y)\right)(x)$, where $\overline{\psi_{G}}=\bar{\pi}^{-1} \overline{\psi_{A}}$. Define $S: X \times X \rightarrow X \times X$ and $S^{\prime}: X \times X \rightarrow X \times X$ via $S(x, y)=\left(x \circ y, y^{-1} * x\right)$ and $S^{\prime}(x, y)=(\phi(y, x), x)$, where $x \circ y$ is defined in such a way that $\phi(y, x)=x^{-1} *((y * x) \circ y)$ holds.

Lemma 3. (i) For an arbitrary bijective cocycle 7-tuple, $(X, S)$ constructed above is a braided nondegenerate set.

(ii) $\left(X, S^{\prime}\right)$ is a derived nondegenerate braided set corresponding to $(X, S)$.

Proof. It is obvious from the definition of a bijective cocycle 7-tuple that $\left(X, S^{\prime}\right)$ is a braided nondegenerate set. We introduced $x \circ y$ in such a way that $\left(X, S^{\prime}\right)$ becomes a derived solution corresponding to $(X, S)$ as long as we are able to show that $(X, S)$ is braided nondegenerate itself. Since $\phi$ is $G$-invariant the argument identical to the one made in the proof of Theorem 2.3 mitigates that for each integer $n \geq 2$ there exists a bijection $J_{n}: X^{n} \rightarrow X^{n}$ such that $J_{n} S_{n}^{i i+1} J_{n}^{-1}=$ $\left(S^{\prime}\right)_{n}^{i i+1}$. This implies that $S$ is bijective and satisfies the braid relation (2.1).

It remains to prove that $(X, S)$ is nondegenerate, i.e. that $x \circ y$ depends bijectively on $y \in X$ for a fixed $x \in X$. For that purpose we show that we can define $g \circ y \in X$ for $y \in X, g \in G$ such that for any $x, y \in X, g, h \in G$ $x \circ y=\overline{\psi_{G}}(x) \circ y$ and $(g h) \circ y=g \circ(h \circ y)$, i.e. $\circ$ is an action of $G$ on $X$.

Let a group homorphism $P: G \rightarrow G \ltimes A$ be defined by the formula $P(g)=$ $(g, \bar{\pi}(g))$ for $g \in G$. Then the map $\rho_{G X}^{*}=\rho_{G A X} P$ is an action of $G$ on $X$. For $g \in G, y \in X$ define

$$
g \circ y=\rho_{G X}^{*}\left(\bar{\pi}^{-1}\left(\rho_{G A}\left(\overline{\psi_{G}}(y)^{-1}\right)(\bar{\pi}(g))\right)\right)(y) .
$$

We want to check $(g h) \circ y=g \circ(h \circ y)$. For notational convience let $\rho_{G A}\left(\overline{\psi_{G}}(y)^{-1}\right)(\bar{\pi}(g))=\overline{\psi_{G}}(y)^{-1} * \bar{\pi}(g)$, i.e. by $*$ we will mean both actions $\rho_{G A}$ and $\rho_{G X}$. Then due to the fact that $P$ is a homomorphism it is enough to verify that

$$
\bar{\pi}^{-1}\left(\overline{\psi_{G}}(h \circ y)^{-1} * \bar{\pi}(g)\right)=\bar{\pi}^{-1}\left(\overline{\psi_{G}}(y)^{-1} * \bar{\pi}(g h)\right)\left(\bar{\pi}^{-1}\left(\overline{\psi_{G}}(y)^{-1} * \bar{\pi}(h)\right)^{-1} .\right.
$$


Since $\bar{\pi}(g h)=\left(h^{-1} * \bar{\pi}(g)\right) \bar{\pi}(h)$,

$$
\begin{aligned}
& \bar{\pi}^{-1}\left(\overline{\psi_{G}}(y)^{-1} * \bar{\pi}(g h)\right)=\bar{\pi}^{-1}\left(\left(\overline{\psi_{G}}(y)^{-1} h^{-1} * \bar{\pi}(g)\right)\left(\overline{\psi_{G}}(y)^{-1} * \bar{\pi}(h)\right)\right) \\
= & \bar{\pi}^{-1}\left(\bar{\pi}^{-1}\left(\overline{\psi_{G}}(y)^{-1} * \bar{\pi}(h)\right) \overline{\psi_{G}}(y)^{-1} h^{-1} * \bar{\pi}(g)\right) \bar{\pi}^{-1}\left(\overline{\psi_{G}}(y)^{-1} * \bar{\pi}(h)\right) .
\end{aligned}
$$

In this way, if we check that

$$
\bar{\pi}^{-1}\left(\overline{\psi_{G}}(y)^{-1} * \bar{\pi}(h)\right) \overline{\psi_{G}}(y)^{-1} h^{-1}=\overline{\psi_{G}}(h \circ y)^{-1}
$$

we conclude that $g \circ(h \circ y)=(g h) \circ y$. Let us rewrite (2.14) as (cf. Lemma 2) $\overline{\psi_{G}}(h \circ y) \bar{\pi}^{-1}\left(\overline{\psi_{G}}(y)^{-1} * \bar{\pi}(h)\right)=h \overline{\psi_{G}}(y)$ and apply $\bar{\pi}$ to it. We get that $(2.14)$ is equivalent to

$\overline{\psi_{A}}\left(\bar{\pi}^{-1}\left(\overline{\psi_{G}}(y)^{-1} * \bar{\pi}(h)\right)^{-1} *(h \circ y)\right)\left(\overline{\psi_{G}}(y)^{-1} * \bar{\pi}(h)\right)=\left(\overline{\psi_{G}}(y)^{-1} * \bar{\pi}(h)\right)\left(\overline{\psi_{A}}(y)\right)$. The last equality follows from $A$-equivariance of $\overline{\psi_{A}}$ since $\bar{\pi}^{-1}\left(\overline{\psi_{G}}(y)^{-1} * \bar{\pi}(h)\right)^{-1} *$ $(h \circ y)=\rho_{A X}\left(\overline{\psi_{G}}(y)^{-1} * \bar{\pi}(h)\right)(y)$ by definition of $h \circ y$. The lemma is proved.

One can combine two actions of $G$ on $X-\rho_{G X}$ and $\rho_{G X}^{*}$ into the action $\rho=\rho_{G X} \times \rho_{G X}^{*}: G \rightarrow \operatorname{Permut}(X) \times \operatorname{Permut}(X)$ of $G$ on $X^{2}$.

Definition 7. We call a bijective cocycle 7-tuple $\left(G, A, X, \rho_{G A}, \rho_{G A X}, \bar{\pi}, \overline{\psi_{A}}\right)$ faithful if $\overline{\psi_{G}}(X)=\bar{\pi}^{-1} \overline{\psi_{A}}(X)$ generates $G$ and the action $\rho: G \rightarrow \operatorname{Permut}(X) \times$ $\operatorname{Permut}(X)$ is faithful.

The following theorem is a characterization of finite braided nondegenerate sets in group theoretical terms. We notice that this result is a generalization of Proposition 2.11 in [ESS].

Theorem 2.7. The construction of Lemma 3 establishes a 1-1 correspondence between nondegenerate braided sets $(X, S)$ and faithful bijective cocycle 7-tuples.

Proof. Having a nondegenerate braided set $(X, S)$ it is straightforward to construct the faithful bijective cocycle 7-tuple. Indeed, following the notations of Theorem 2.6 we put $G=G_{X} / \Gamma, A=A_{X} / \pi(\Gamma)$ then $G$ acts faithfully on $X^{2}$ and we have a faithful cocycle 7-tuple. Conversely, if we have a faithful 7-tuple we can construct a nondegenerate braided set by Lemma 3 . Let $G_{X}$ be the structure group of so constructed nondegenerate braided set. It follows from (2.14) that there is a group homomorphism $Q: G_{X} \rightarrow G$ such that $Q \psi_{G}=\overline{\psi_{G}}$. Since $\overline{\psi_{G}}(X)$ generates $G, Q$ is surjective, and since $G$ acts faithfully on $X^{2}$, $\operatorname{Ker}(Q)=\Gamma$, i.e. $G_{X} / \Gamma$ is isomorphic to $G$.

2.3. Injective solutions. In this section we talk about most tractable braided nondegenerate sets - injective solutions.

Definition 8. We call a braided nondegenerate set $(X, S)$ an injective solution if the map $\psi_{G}: X \rightarrow G_{X}$ is injective.

In [LYZ] the authors noticed that in the absence of involutivity the natural map $\psi_{G}: X \rightarrow G_{X}$ is not obviously injective which creates difficulties in characterization of solutions. It turned out that injectivity may indeed fail (see examples below). This motivates Definition 8. 
Lemma 4. A nondegenerate braided set $(X, S)$ is injective if and only if its derived solution $\left(X, S^{\prime}\right)$ is injective.

Proof. The statement of Theorem 2.5 implies that injectivity of the map $\psi_{A}$ : $X \rightarrow A_{X}$ is equivalent to injectivity of $\psi_{G}: X \rightarrow G_{X}$. Since $A_{X}$ is the structure group of the derived solution Lemma 4 is proved.

The importance of injective solutions is in the fact that their properties and group-theoretical characterization are very similar to that of involutive solutions [ESS].

Theorem 2.8. (i) Let a group $G$ act on a group $A$ by $\rho_{G A}: G \rightarrow \operatorname{Aut}(A)$ such that the bijective map $\pi: G \rightarrow A$ is a 1-cocycle. Then any $G \ltimes A$-invariant subset $X \subset A$ has a natural structure of a nondegenerate braided injective set given by

$$
S(x, y)=\left(\pi\left(\pi^{-1}(x) \pi^{-1}(y)\left(\pi^{-1}\left(\rho\left(\pi^{-1}(y)^{-1}\right)(x)\right)\right)^{-1}\right), \rho\left(\pi^{-1}(y)^{-1}\right)(x)\right),
$$

for $x, y \in X$.

(ii) Any nondegenerate braided injective set can be obtained by the method just described.

Proof. To prove (i) we can use Lemma 3. Indeed, we have the following bijective cocycle 7-tuple: $G, A, X, \bar{\pi}=\pi, \rho_{G A}$ as given; $\rho_{G A X}$ is induced from the adjoint action of $G \ltimes A$ on its subgroup $A, \overline{\psi_{A}}=i d_{X}$. It is straightforward to check (cf. (2.14)) that the map $S$ constructed in Lemma 3 coincides with the map $S$ given by formula $(2.15)$. So it remains to prove that the set $(X, S)$ is injective. Let $G_{X}$ be its structure group. Arguing as in the proof of Theorem 2.7 we see that there is a group homomorphism $Q: G_{X} \rightarrow G$ such that $\pi Q \psi_{G}=i d_{X}$. This implies that $\psi_{G}$ is injective.

Conversely, if $(X, S)$ is an injective nondegenerate braided set then $X=$ $\psi_{A}(X)$ is a $G_{X}$-invariant (w.r.t. $*$ - action) subset in $A_{X}$. Note that this subset is automatically $G_{X} \ltimes A_{X}$-invariant. Recall that $S$ is given by the formula $S(x, y)=\left(x \circ y, y^{-1} * x\right)$ for $x, y \in X$ according to notations after Theorem 2.2. Let us make sure that the construction of Theorem 2.8 yields the same map $S$ we already have. Indeed, for $x, y \in \psi_{A}(X)=X, \pi^{-1}(y)^{-1} * x=\psi_{G}(y)^{-1} * x=y^{-1} * x$ and $\pi\left(\pi^{-1}(x) \pi^{-1}(y)\left(\pi^{-1}\left(\pi^{-1}(y)^{-1} * x\right)\right)^{-1}\right)=\pi\left(\psi_{G}(x) \psi_{G}(y) \psi_{G}\left(y^{-1} * x\right)^{-1}\right)=$ $\pi\left(\psi_{G}(x \circ y)\right)=\psi_{A}(x \circ y)=x \circ y$. The theorem is proved.

Lemma 4 implies that injectivity of a given solution is determined by the properties of the function $\phi: X \times X \rightarrow X$. In particular, for a symmetric set $(X, S), \phi(y, x)=y$ and $A_{X}$ is the free abelian group generated by $X$. Hence, symmetric sets are injective. We don't know any easy way to check that a given function $\phi(y, x)$ corresponds to an injective solution. While an injectivity criterion is provided by Theorem 2.9, we give two simple necessary conditions below that are in many cases sufficient to check that a given solution is not injective. 
Lemma 5. If $(X, S)$ is an injective braided set then

(a) $\phi(x, x)=x$ for all $x \in X$,

(b) a pair $(y, x) \in X \times X$ satisfies $\phi(y, x)=y$ if and only if $\phi(x, y)=x$.

Proof. Suppose $(X, S)$ is injective. The group $A_{X}$ is generated by the elements of $X$ subject to relations $\phi(y, x) \bullet y=y \bullet x$ for $x, y \in X$. Consequently $\psi_{A}(\phi(x, x)) \bullet \psi_{A}(x)=\psi_{A}(x) \bullet \psi_{A}(x)$ and $\psi_{A}(\phi(x, x))=\psi_{A}(x)$ in $A_{X}$. Since $\psi_{A}: X \rightarrow A_{X}$ is injective $\phi(x, x)=x$ on $X$. Now, assume that $\phi(y, x)=y$. Then $\psi_{A}(y) \bullet \psi_{A}(x)=\psi_{A}(x) \bullet \psi_{A}(y)$. On the other hand, $\psi_{A}(\phi(x, y)) \bullet \psi_{A}(y)=$ $\psi_{A}(y) \bullet \psi_{A}(x)$, therefore $\psi_{A}(\phi(x, y))=\psi_{A}(x)$ and $\phi(x, y)=x$. The lemma is proved.

Example. Let $c, b \in \operatorname{Permut}(X)$. Define $S: X \times X \rightarrow X \times X$ by the formula $S(x, y)=(b y, c x)$. It is easy to see that $(X, S)$ is a nondegenerate braided set if $b c=c b$. We claim that this solution is injective if and only if $c b=i d_{X}$. Indeed, suppose the solution is injective, then by Lemma $5 \phi(x, x)=x$, i.e. $x^{-1} *((x * x) \circ x)=c b x=x$. Therefore $c b=i d_{X}$. Conversely, if $c b=i d_{X}$ then $(X, S)$ is symmetric and hence injective.

We remark that with each nondegenerate braided set we associated two actions of the group $G_{X}(*, \circ)$ and an action of $A_{X}$ (via $\phi(x, y)$, which is a $\circ$ action of the derived solution) on $X$. In particular, the latter action allows us to construct a finite group $A_{X}^{0} \subset \operatorname{Permut}(X)$, as the image of $A_{X}$ under that action, and a surjective homomorphism $p: A_{X} \rightarrow A_{X}^{0}$. Define $M_{X}$ - a module over $A_{X}^{0}$ generated by $v_{x}, x \in X$ subject to relations

$$
p(y)^{-1} v_{x}+v_{y}=p(x)^{-1} v_{\phi(y, x)}+v_{x} .
$$

By construction we have a natural map $\psi_{M}: X \rightarrow M_{X}$ given by $x \rightarrow v_{x}$. It turns out that injectivity of $\psi_{M}$ is equivalent to injectivity of $\psi_{G}$.

Theorem 2.9. (i) There exists a unique 1-cocycle $\theta: A_{X} \rightarrow M_{X}$ such that $\theta \psi_{A}=\psi_{M}$, where $A_{X}$ acts on $M_{X}$ via $p$.

(ii) $\theta$ is injective on $\psi_{A}(X)$.

Proof. Statement (i) is clear from definitions of $A_{X}$ and $M_{X}$. Let us show that (ii) holds. Let $\operatorname{Ker}(p)=\Gamma_{A}$, i.e. $A_{X}^{0}=A_{X} / \Gamma_{A}$. Let $x_{1}, x_{2} \in \psi_{A}(X), x_{1} \neq x_{2}$. We want to show that $\theta\left(x_{1}\right) \neq \theta\left(x_{2}\right)$ in $M_{X}$. Fix a character $\xi: \Gamma_{A} \rightarrow \mathbb{C}^{*}$. Define a vector space

$$
V_{\xi}=\left\{f: A_{X} \rightarrow \mathbb{C} \mid f(a \bullet \gamma)=f(a) \xi(\gamma), \gamma \in \Gamma_{A}\right\}
$$

$V_{\xi}$ clearly has an $A_{X}$-module structure defined as $(b f)(a)=f\left(b^{-1} \bullet a\right)$, where $f \in V_{\xi}, b, a \in A_{X}$. Choose a lifting $g: A_{X}^{0} \rightarrow A_{X}$ (as a set only). Then $V_{\xi}$ is identified with $\operatorname{Fun}\left(A_{X}^{0}, \mathbb{C}\right)$, the space of functions on $A_{X}^{0}$ via $\left.f \rightarrow f\right|_{g\left(A_{X}^{0}\right)}$. This space has a basis $\delta_{a}, a \in A_{X}^{0}$ such that $\delta_{a}(b)=0$ if $a \neq b$ and $\delta_{a}(a)=1$. Let us define $\varepsilon: \psi_{A}(X) \rightarrow F u n\left(A_{X}^{0}, \mathbb{C}\right)$ by the formula $x \delta_{a}=\delta_{p(x) \bullet a} \varepsilon(x)(a)$ for $x \in \psi_{A}(X), a \in A_{X}^{0}$. We can always choose $\xi$ and $g$ such that $\varepsilon\left(x_{1}\right) \neq \varepsilon\left(x_{2}\right)$. Indeed, if $p\left(x_{1}\right)=p\left(x_{2}\right)$ it suffices to choose $\xi$ such that $\xi\left(x_{1} \bullet x_{2}^{-1}\right) \neq 1$ for any 
lifting $g$. If $p\left(x_{1}\right) \neq p\left(x_{2}\right)$ then for any character $\xi \neq 1$ there is a lifting $g$ such that $\varepsilon\left(x_{1}\right) \neq \varepsilon\left(x_{2}\right)$. Moreover, since $x \bullet y=\phi(y, x) \bullet x$ for $x, y \in \psi_{A}(X)$ and

$$
x y \delta_{a}=x \delta_{p(y) \bullet a} \varepsilon(y)(a)=\delta_{p(x) \bullet p(y) \bullet a} \varepsilon(x)(p(y) \bullet a) \varepsilon(y)(a),
$$

we get that

$$
\varepsilon(x)(p(y) \bullet a) \varepsilon(y)(a)=\varepsilon(\phi(y, x))(p(x) \bullet a) \varepsilon(x)(a) .
$$

In this way, we have the equality $\left(p(y)^{-1} \varepsilon(x)\right) \varepsilon(y)=\left(p(x)^{-1} \varepsilon(\phi(y, x))\right) \varepsilon(x)$ in $F u n\left(A_{X}^{0}, \mathbb{C}\right)$. Hence we can construct an $A_{X}^{0}$-homomorphism from $M_{X}$ to $F u n\left(A_{X}^{0}, \mathbb{C}\right)$ given by $v_{z} \rightarrow \varepsilon\left(\psi_{A}(z)\right), z \in X$. But $\varepsilon\left(x_{1}\right) \neq \varepsilon\left(x_{2}\right)$ thus $\theta\left(x_{1}\right) \neq$ $\theta\left(x_{2}\right)$.

Corollary 1. The map $\psi_{M}: X \rightarrow M_{X}$ is injective if and only if $(X, S)$ is an injective solution.

2.4. Rank of the structure group. In this section we show how to compute the rank of the structure group $G_{X}$ for a finite nondegenerate braided set $(X, S)$.

Definition 9. (i) The rank of a group $G$ having an abelian subgroup of finite index $\Gamma$ is defined as the rank of $\Gamma$.

(ii) We define the rank of a finite nondegenerate braided set $(X, S)$ to be the rank of its structure group $G_{X}$.

Clearly the above definition doesn't depend on the choice of $\Gamma$, for any two abelian subgroups of finite index $\Gamma_{1}, \Gamma_{2}$ have the same rank that is equal to the rank of their intersection, which has finite index in each of them.

Lemma 6. The rank of a solution $(X, S)$ is equal to the rank of the derived solution $\left(X, S^{\prime}\right)$.

Proof. According to statement (i) of Theorem 2.6 abelian subgroups $\Gamma$ and $\pi(\Gamma)$ of finite indexes in $G_{X}$ and $A_{X}$ are isomorphic, thus $G_{X}$ and $A_{X}$ have the same rank. The lemma is proved.

We aim to compute the rank of $A_{X}$. Note that defining relations in group $A_{X}$ can be rewritten as

$$
\phi(y, x)=x \bullet y \bullet x^{-1} .
$$

Note that since $S(x, y)=(\phi(y, x), x)$ gives rise to a nondegenerate braided set, $\phi(, x)$ can be extended to the action of $A_{X}$ on $X$. Introduce an equivalence relation $\backsim$ on $X$ such that the orbits of the above action become equivalence classes. Namely, consider the minimum equivalence relation $\backsim$ such that $y \backsim$ $\phi(y, x)$ for $x, y \in X$.

Theorem 2.10. The rank of group $A_{X}$ is equal to the number of equivalence classes with respect to equivalence relation $\backsim$ on $X$. 
The proof relies on the following lemma. Let $H_{1}, H_{2}, H_{3}$ be three groups, such that there is an exact sequence

$$
1 \rightarrow H_{1} \rightarrow H_{2} \rightarrow H_{3} \rightarrow 1
$$

and $H_{1}$ is cenral in $H_{2}$.

Lemma 7 ([CR]). There exists an exact sequence (The Hochschild - Serre sequence) (2.17) for any abelian group $B$, where $H^{2}\left(H_{3}, B\right)$ stands for the second cohomology group of $\mathrm{H}_{3}$ with coefficients in $B$.

$$
1 \rightarrow \operatorname{Hom}\left(H_{3}, B\right) \rightarrow \operatorname{Hom}\left(H_{2}, B\right) \rightarrow \operatorname{Hom}\left(H_{1}, B\right) \rightarrow H^{2}\left(H_{3}, B\right) .
$$

Proof of Theorem 2.10. Let us use the sequence 2.17 in the following situation: $H_{2}=A_{X}, H_{1} \subset \pi(\Gamma) \subset A_{X}$ is the free abelian group of rank $r$ of finite index in $\pi(\Gamma), H_{3}=H_{2} / H_{1}$ - a finite group, $B=\mathbb{C}^{*}$. Notice that $\pi(\Gamma)$ is central in $A_{X}$ therefore $H_{1}$ is central in $H_{2}$. Then, since both $\operatorname{Hom}\left(H_{3}, B\right)$ and $H^{2}\left(H_{3}, B\right)$ are finite groups and $\operatorname{Hom}\left(H_{1}, B\right)=\left(\mathbb{C}^{*}\right)^{r}$, the dimension of $\operatorname{Hom}\left(H_{2}, B\right)=$ $\operatorname{Hom}\left(A_{X}, \mathbb{C}^{*}\right)$ is equal to $r$, the rank of $A_{X}$. On the other hand, it is clear from formula (2.16) that $\operatorname{Hom}\left(A_{X}, \mathbb{C}^{*}\right)=\left(\mathbb{C}^{*}\right)^{k}$, where $k$ is the number of equivalence classes in $X$. The theorem is proved.

Corollary 1. The rank of any solution $(X, S)$ is less or equal than $n$, the number of elements in $X$, with the equality taking place if and only if $(X, S)$ is symmetric.

Proof. It is clear that if rank of $A_{X}$ is equal to $n$ then equivalence relation $\backsim$ on $X$ is trivial, $\phi(y, x)=y$ and the map $\psi_{A}: X \rightarrow A_{X}$ is injective. This immediately implies that the pair $(X, S)$ is symmetric.

Example. Consider a permutation solution $S(x, y)=(b x, c y), b, c \in$ $\operatorname{Permut}(X), b c=c b$. It is easy to check that $\phi(y, x)=b c y$, hence $y \sim(b c)^{m} y$ for any integer $\mathrm{m}$. The rank of the permutation solution is equal to the number of equivalence classes with respect to this relation, which, in turn, is equal to the number of independent cyclic permutations in canonical decomposition of $b c$ (counting cyclic permutations of length 1 ).

\section{Linear and affine solutions}

3.1. Linear braided sets. In this section we will look for nondegenerate braided sets of the following form: $X$ is an abelian group, and $S$ is an affine linear transformation of $X \times X$. Such braided sets will be called affine solutions. Considering affine solutions was motivated by the results in [ESS].

We will start with considering a special case, when $S$ is an automorphism of $X \times X$. In this case, an affine solution will be called a linear solution. For a linear solution, $S$ has the form

$$
S(x, y)=(a x+b y, c x+d y), a, b, c, d \in \operatorname{End}(X) .
$$


It is easy to check that for $S$ of the form (3.1) the nondegeneracy is equivalent to invertibility of both $b$ and $c$ while braid relation is equivalent to the equations $[\mathrm{Hi}]$

$$
\begin{aligned}
& a(1-a)=b a c, \quad d(1-d)=c d b, \\
& a b=b a(1-d), \quad c a=(1-d) a c, \quad d c=c d(1-a), \\
& b d=(1-a) d b, \quad c b-b c=a d a-d a d .
\end{aligned}
$$

Lemma 8. Braided nondegenerate linear sets $(X, S)$ are in 1-1 correspondence with the quadruples $(a, b, d, s) \in \operatorname{End}(X)^{4}$ such that:

(i) $1-a, 1-d, b, 1+s$ are invertible,

(ii) $s$ commutes with $a, b, d$ and $s a=s d=0$,

(iii) $b d b^{-1}=(1-a) d, b^{-1} a b=a(1-d)$. The 1-1 correspondence is given via the formula

$$
b c=(1-d+a d)(1-a)+s .
$$

Proof. Suppose $(a, b, c, d)$ solves (3.2)-(3.4). Note that the first of equations (3.4) implies that $b d b^{-1}=(1-a) d$, therefore $b(1-d) b^{-1}=1-d+a d$. Moreover, if we multiply the first equation of (3.3) by $b^{-1}$ on the right we get that $a=$ $b a b^{-1} b(1-d) b^{-1}$ thus relations (3.6) hold. Similarly from equations two and three of (3.3) we obtain relations (3.7).

$$
\begin{aligned}
& b a b^{-1}(1-d+a d)=a, b d b^{-1}=(1-a) d, \\
& c a c^{-1}=(1-d) a, c d c^{-1}(1-a+d a)=d .
\end{aligned}
$$

Above formulas show how to conjugate the elements of subalgebra generated by $a$ and $d$ by elements $b, c$ and their products. We define $s$ from the relation (3.5). Notice that $s a=s d=0$. Indeed, multiplying (3.5) by $a$ on the right and using first of relations (3.2) and second of relations (3.3) we get that

$$
s a=b c a-(1-d+a d)(1-a) a=b(1-d) a c-(1-d+a d) b a c .
$$

Since according to $(3.6) b(1-d) b^{-1}=1-d+a d$ we see that $b(1-d) a c-(1-$ $d+a d) b a c=0$. Similarly $a s=0$. The last of equations (3.4) imply that

$$
c b=(1-a+d a)(1-d)+s .
$$

Multiplying the relation (3.8) just obtained by $d$ we get that $s d=d s=0$. Now we have everything to show that $1-a, 1+s$ and $1-d$ are invertible. Indeed, $b, c$ are invertible because of nondegeneracy of $(X, S)$. Since

$$
\begin{aligned}
& b c=(1-d+s+a d)(1-a)=(1-a)(1-d+s+d a), \\
& b c=(1-d+a d)(1-a+s)=(1-a+s)(1-d+a d),
\end{aligned}
$$

and

$$
c b=(1-a+s+d a)(1-d)=(1-d)(1-a+s+a d),
$$

we conclude that $1-a, 1-a+s$ and $1-d$ have right and left inverses and hence invertible. But $1-a+s=(1-a)(1+s)$, thus $1+s$ is invertible. Let us show 
that $s$ commutes with $c$. We conjugate relation (3.5) by $c$ and use relations (3.7) to conclude that

$$
c b=(1-a+d a)(1-d)+c s c^{-1} .
$$

Comparing (3.8) to (3.9) we get that $s=c s c^{-1}$, i.e. $s$ commutes with $c$. Now, relation (3.5) implies that $s$ commutes with $b$ as well. In this way, starting from $(a, b, c, d)$, a solution to (3.2)-(3.4) we constructed a quadruple $(a, b, d, s)$ satisfying conditions of the lemma. Conversely, let us assume we start with $(a, b, d, s)$. Define $c$ from the formula (3.5). Since $1-d+a d=b(1-d) b^{-1}$ is invertible, $c$ is invertible as well. It is straightforward to check that so defined $(a, b, c, d)$ satisfy the braid relations (3.2)-(3.4), and that $S: X \times X \rightarrow X \times X$ is bijective. The lemma is proved.

3.2. Injective linear solutions. It turns out that injective linear solutions are easy to characterize.

Theorem 3.1. A linear nondegenerate braided set of the form (3.1) is injective iff $b c=(1-d+a d)(1-a)$ or, in the language of Lemma $8, s=0$.

Proof. Assume that $(X, S)$ is injective. Then, by Lemma $5 \phi(x, x)=x$ on $X$. In the linear case it is easy to compute $\phi(y, z)$ explicitly:

$\phi(y, z)=c((y * z) \circ y)+d z=c(a(y * z)+b y)+d z=c a c^{-1}(z-d y)+c b y+d z$.

According to relation $(3.8), c b=(1-a+d a)(1-d)+s$. Plugging it into formula for $\phi(y, z)$ and using (3.7) we conclude that

$$
\phi(y, z)=(1-(1-d)(1-a)) z+((1-d)(1-a)+s) y .
$$

Condition $\phi(x, x)=x$ immediately implies that $s=0$.

Conversely, assume that $s=b c-(1-d+a d)(1-a)=0$. Let us show that $\psi_{A}: X \rightarrow A_{X}$ is injective. The group $A_{X}$ is generated by elements of $X$ subject to relations

$$
\phi(y, z) \bullet z=z \bullet y \text {, where } y, z \in X \text { and } \phi(y, z)=z^{-1} *((y * z) \circ y) .
$$

Denoting $K=(1-d)(1-a)$ we see that the group $A_{X}$ is given by relations $((1-K) z+K y) \bullet z=z \bullet y$. By Lemma $8, K$ is invertible. Let us now define the action of $A_{X}$ on $X$. We let the elements of generating set $\psi_{A}(X)$ act with $K$ on $X$ and then extend this action to arbitrary elements of $A_{X}$. Consider a semidirect product $A_{X} \ltimes X$ with respect to this action, and define an embedding $J: X \rightarrow A_{X} \ltimes X$ given by the formula $J(x)=\left(\psi_{A}(x), x\right)$. We notice that $J((1-K) z+K y) J(z)=J(z) J(y)$ in $A_{X} \ltimes X$. Indeed,

$$
\begin{aligned}
J((1-K) z+K y) J & (z) \\
& =\left(\psi_{A}((1-K) z+K y),(1-K) z+K y\right)\left(\psi_{A}(z), z\right) \\
& =\left(\psi_{A}((1-K) z+K y) \bullet \psi_{A}(z), K^{-1}((1-K) z+K y)+z\right) \\
= & \left(\psi_{A}(z) \bullet \psi_{A}(y), K^{-1} z+y\right)=J(z) J(y) .
\end{aligned}
$$


So, $J$ can be extended to a homomorphism $\hat{J}: A_{X} \rightarrow A_{X} \ltimes X$ such that $\operatorname{Pr} \hat{J} \psi_{A}=i d$, where $\operatorname{Pr}: A_{X} \ltimes X \rightarrow X$ stands for the projection to the second component. Therefore, the map $\psi_{A}: X \rightarrow A_{X}$ is injective. The theorem is proved.

Corollary 1. (i) Let $(X, S)$ be a nondegenerate braided linear set of the form (3.1). Then the pair $(X, \hat{S})$ with $\hat{S}: X \times X \rightarrow X \times X$ given by $\hat{S}(x, y)=$ $(a x+b y, c x+(d-s) y)$, where $s$ is defined in 3.5, is an injective solution.

(ii) Suppose $(X, S)$ is an injective linear solution and $s: X \rightarrow X$ satisfies $s a=a s=0, s b=b s, s d=d s=-s^{2}, s c=c s$. Then, $(X, \breve{S})$ with $\breve{S}: X \times X \rightarrow$ $X \times X$ given by $\breve{S}(x, y)=(a x+b y, c x+(d+s) y)$ is a nondegenerate braided set that corresponds under the correspondence of Lemma 8 to the quadruple $(a, b, d, s)$.

Proof. (i) It is easy to check $(X, \hat{S})$ is a braided nondegenerate set by directly checking relations (3.2) - (3.4). Since $s$ commutes with everything and $s a=$ $s d=0$ the above task is pretty simple. Also, it is obvious that $b c=(1-(d-$ $s)+a(d-s))(1-a)$, thus by Theorem $3.1(X, \hat{S})$ is injective. Proof of (ii) is similar to (i) and is left to the reader.

Examples. 1. Consider the linear solution $S(x, y)=(c y, b x)$ with $c, b$ being linear automorphisms of $X$ subject to $c b=b c$. We see that $\mathrm{s}=\mathrm{bc}-1$, therefore $(X, \hat{S})$ with $\hat{S}(x, y)=(c y, b x+(1-b c) y)$ is an injective solution. We obtain the same solution by a different method in Example 2 at the end of this section.

2. It was shown in [ESS] that symmetric nondegenerate linear solutions of the form 3.1 are given as the solutions to the following equations: $b a b^{-1}=$ $\frac{a}{a+1}, c=b^{-1}\left(1-a^{2}\right), d=\frac{a}{a-1}$ with $b, c$ being invertible. In particular a large class of solutions of this kind considered corresponded to nilpotent $a$, i.e. there was $n$ such that $a^{n}=0$. Define $s=m a^{n-1}, m$ being any integer. It is easy to see that so defined $s$ satisfies conditions of Corollary 1 (ii) hence $S^{\prime \prime}(x, y)=\left(a x+b y, c x+\left(d+m a^{n-1}\right) y\right)$ is a nondegenerate braided set, which is not symmetric and not injective unless $m a^{n-1}=0$.

Theorem 3.2. Linear braided nondegenerate injective sets $(X, S)$ on an abelian group $X$ are in 1-1 correspondence with triples $(a, b, d)$ of endomorphisms of $X$ such that $b, 1-a, 1-d$ are invertible and $b d b^{-1}=(1-a) d, b^{-1} a b=a(1-d)$.

Proof. Straightforward application of Lemma 8 and Theorem 3.1.

Corollary 1. Linear braided nondegenerate injective sets $(X, S)$ on an abelian group $X$ are in 1-1 correspondence with triples $(p, q, z)$ of automorphisms of $X$ such that $p q=q p$ and $z^{2}-z(p+q)+p q=0$.

The statement of Corollary 1 follows from Theorem 3.2 via change of variables $p=b^{-1}, q=(1-a)(1-d) b^{-1}, z=(1-a) b^{-1}$.

Examples. 1. If $(X, S)$ is unitary then $(1-a)(1-d)=1$ and therefore $p=q$. In this way, nondegenerate unitary linear braided sets are characterized as 
representations of algebra generated by invertible $p, z$ subject to $z^{2}-2 z p+p^{2}=$ 0 .

2. Put $z=p$, then $q$ can be anything as long as it is invertible and $p q=q p$. Correspondingly, $a=0, b=p^{-1}, 1-d=p^{-1} q, c=q$. The map $S$ is defined in the following way: $S(x, y)=\left(p^{-1} y, q x+\left(1-p^{-1} q\right) y\right)$. Similarly if we let $z=q$ we obtain the solution given by the formula: $S(x, y)=\left(p y+\left(1-q^{-1} p\right) x, q^{-1} x\right)$.

3. Let $\epsilon_{1}$ and $\epsilon_{2}$ be two nilpotent operators on $X$ of order two, i.e. $\epsilon_{1}^{2}=\epsilon_{2}^{2}=0$. Then we let $p=1+\epsilon_{1}, q=1-\epsilon_{1}, z=1+\epsilon_{2}$. It is easy to check that so defined $p$ and $q$ commute and that $z^{2}-z(p+q)+p q=0$. We recover $a, b, c, d$ from $\epsilon_{1}$ and $\epsilon_{2}$ via the formulas $b=1-\epsilon_{1}, a=\epsilon_{1}-\epsilon_{2}+\epsilon_{2} \epsilon_{1}, d=1-\left(1+\epsilon_{1}\right)\left(1-\epsilon_{2}\right)\left(1-2 \epsilon_{1}\right)$, $c=\left(1+\epsilon_{1}\right)\left(1-\epsilon_{2}\right)\left(1-\epsilon_{1}\right)\left(1+\epsilon_{2}\right)\left(1-\epsilon_{1}\right)$. In particular, if $\epsilon_{1} \epsilon_{2}=\epsilon_{2} \epsilon_{1}$ the corresponding linear solution has the form:

$$
S(x, y)=\left(\left(\epsilon_{1}-\epsilon_{2}+\epsilon_{2} \epsilon_{1}\right) x+\left(1-\epsilon_{1}\right) y,\left(1-\epsilon_{1}\right) x+\left(\epsilon_{1}+\epsilon_{2}-\epsilon_{2} \epsilon_{1}\right) y\right)
$$

4. Suppose $X$ is a n-dimensional vector space and $p, q$ are invertible operators on it having $2 n$ distinct eigenvalues. Let $\left(v_{1} \ldots v_{n}\right)$ be the basis of $X$ in which both $p$ and $q$ are diagonalized, i.e. $p v_{i}=p_{i} v_{i}, q v_{i}=q_{i} v_{i}$. Then $z v_{i}=z_{i} v_{i}$, where for each $i$ either $z_{i}=p_{i}$ or $z_{i}=q_{i}$. Indeed, $0=\left(z^{2}-z(p+q)+p q\right) v_{i}=$ $\left(z-p_{i}\right)\left(z-q_{i}\right) v_{i}$. The vector subspace of $X$ generated by applying $z$ to $v_{i}(i$ is fixed) is annihilated by $\left(z-p_{i}\right)\left(z-q_{i}\right)$ therefore it has a basis of $p_{i}$ and $q_{i}$ eigenvectors of $z$. Therefore, it has to be one dimensional, i.e. $z v_{i}=p_{i} v_{i}$ or $z v_{i}=q_{i} v_{i}$. It is also easy to see that $z$ given by diagonal matrix in $\left(v_{1} \ldots v_{n}\right)$ basis with $p_{i}$ or $q_{i}$ entries on the $i$-th place does satisfy $z^{2}-z(p+q)+p q=0$ on $X$.

3.3. Affine solutions. In this section we talk about general affine solutions on an abelian group $X$.

Definition 10. The solution (braided, nondegenerate) $(X, S)$ of the form (3.11) is called affine.

$$
S(x, y)=(a x+b y+z, c x+d y+t), \quad a, b, c, d \in \operatorname{End}(X), t, z \in X .
$$

Lemma 9. The pair $(X, S)$ of the form (3.11) is a solution if and only if (3.2)(3.4) and (3.12) hold. Therefore, any affine solution gives rise to a linear solution $\left(X, S^{*}\right)$ with $S^{*}(x, y)=(a x+b y, c x+d y)$.

(3.12) $\quad c d z+d t=0, a z+b a t=0,(c+d-a d-1) z+(d a+1-a-b) t=0$.

Proof. Straightforward.

Definition 11. We call $\left(X, S^{*}\right)$ from Lemma 9 the linear part of an affine solution $(X, S)$. Conversely, we call $(X, S)$ an affine solution associated with $\left(X, S^{*}\right)$.

Theorem 3.3. (i) Let $\left(X, S^{*}\right)$ be a linear braided nondegenerate set, $S^{*}(x, y)=$ $(a x+b y, c x+d y)$. Then, $(X, S)$ given by $(3.11)$ is an affine solution associated with $\left(X, S^{*}\right)$ if and only if $t=-c(1-a)^{-1} z+k$ and $a k=d k=0,(b-1) k=s z$, where $s$ is defined in (3.5). 
(ii) An affine solution $(X, S)$ is injective if and only if its linear part is injective and $k=0$ in the above characterization. In this way, injective affine solutions associated with a given injective linear solution $\left(X, S^{*}\right)$ are in 1-1 correspondence with elements $z \in X, t$ being given by $t=-c(1-a)^{-1} z$.

Proof. Let us proof part (i). Note that since $c d z+d t=d\left(c(1-a)^{-1} z+t\right)$ and $\mathrm{az}+\mathrm{bat}=a\left(z+(1-a) c^{-1} t\right)$ by equations (3.2)-(3.3) we can rewrite two of the relations $(3.12)$ as

$$
d\left(c(1-a)^{-1} z+t\right)=0, a\left(z+(1-a) c^{-1} t\right)=0 .
$$

Therefore, if we define $k$ from the relation

$$
t=-c(1-a)^{-1} z+k,
$$

we see using (3.2) that $a k=d k=0$. Now, (3.7) implies $c(1-a)^{-1} c^{-1}=$ $(1-a+d a)^{-1}$ thus we can transform (3.13) into $(1-a+d a) t=-c z+(1-a+d a) k=$ $-c z+k$. In this way, we rewrite last of the relations (3.12) as

$$
(d-a d-1) z-b t+k=0
$$

If we substitute $t$ from (3.13) into (3.14) and use (3.5) we get that $(b-1) k=$ sz. Part (i) is proved. In order to prove part (ii) we compute the function $\phi: X \times X \rightarrow X$. Define by $\phi^{*}$ the corresponding function $\phi$ of the linear part $\left(X, S^{*}\right)$. Then, it is easy to check that $\phi(y, x)=\phi^{*}(y, x)+k$. Now, assume that $(X, S)$ is injective. Then by Lemma $5 \phi(x, x)=x$, hence $\phi^{*}(x, x)=x$ and $k=0$. As we saw in the proof of Theorem $3.1 \phi^{*}(x, x)=x$ implies that $s=0$ and thus $\left(X, S^{*}\right)$ is injective. Conversely, if $\left(X, S^{*}\right)$ is injective and $k=0$ then $\phi(y, x)=\phi^{*}(y, x)$ and hence the derived solution of $(X, S)$ coincides with the derived solution of $\left(X, S^{*}\right)$ and hence is injective. Theorem is proved.

\section{Acknowledgments}

The author is thankful to his advisor Pavel Etingof for a valuable exchange of ideas and guidance throughout the work on the paper, and to Lu, Yan, Zhu for making their work available before publication.

\section{References}

[Dr] V. G. Drinfeld, On some unsolved problems in quantum group theory, Lecture Notes in Math 1510, 1-8.

[ESS] P. Etingof, T. Schedler, and A. Soloviev, Set-theoretical solutions to the quantum YangBaxter equation, Duke Math. J. 100, 169-209, q-alg/9801047.

[CR] C. W. Curtis and I. Reiner, Methods of representation theory. Vol. I. With applications to finite groups and orders, Pure and Applied Mathematics, A Wiley-Interscience Publication, John Wiley \& Sons, New York, 1981.

[Hi] J. Hietarinta, Permutation-type solutions to the Yang-Baxterand other simplex equations, J. Phys. A. 30, 4757-4771, q-alg/9702006.

[LYZ] J.-H. Lu, M. Yan, and Y.-C. Zhu, On set-theoretical Yang-Baxter equation, to appear in Duke Math. J. 2000.

Department of Mathematics, Mit, Cambridge, MA 02139, USA.

E-mail address: sashas@math.mit.edu 